\title{
Robot-Mediated Interviews: Do robots possess advantages over human interviewers when talking to children with special needs?
}

Luke Jai Wood, Kerstin Dautenhahn, Hagen Lehmann, Ben Robins, Austen Rainer, Dag Sverre Syrdal

Adaptive Systems Research Group, University of Hertfordshire, United Kingdom

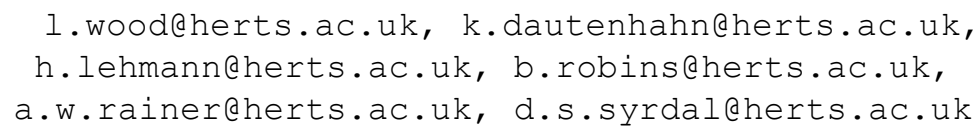

\begin{abstract}
Children that have a disability are up to four times more likely to be a victim of abuse than typically developing children. However, the number of cases that result in prosecution is relatively low. One of the factors influencing this low prosecution rate is communication difficulties. Our previous research has shown that typically developing children respond to a robotic interviewer very similar compared to a human interviewer. In this paper we conduct a follow up study investigating the possibility of Robot-Mediated Interviews with children that have various special needs. In a case study we investigated how 5 children with special needs aged 9 to 11 responded to the humanoid robot KASPAR compared to a human in an interview scenario. The measures used in this study include duration analysis of responses, detailed analysis of transcribed data, questionnaire responses and data from engagement coding. The main questions in the interviews varied in difficulty and focused on the theme of animals and pets. The results from quantitative data analysis reveal that the children interacted with KASPAR in a very similar manner to how they interacted with the human interviewer, providing both interviewers with similar information and amounts of information regardless of question difficulty. However qualitative analysis suggests that some children may have been more engaged with the robotic interviewer.
\end{abstract}

Keywords: Humanoid robots, interviews, children, human-robot interaction, disclosure, interaction dynamics, social interaction

\section{Introduction}

In recent years research investigating how robots can serve as educational, therapeutic and assistive tools has steadily increased [1, 2]. One area of social robotics investigating social mediation for children with special needs is particularly promising, and indicates that children with special needs such as autism often respond well to robots [3-5]. A new emergent application area of social robotics "Robot- 
Mediated Interviews", investigates how robots could be used to communicate with children in an interview scenario. Studies suggest that typically developing children respond well to a robot in an interview scenario [6-9], however, to date there has been very little research investigating how children with special needs would respond to a robot in an interview scenario. This paper endeavours to investigate on a case study basis, how children with a variety of special needs respond to a robot in an interview scenario compared to a human interviewer. Children with special needs such as autism can sometimes be more difficult to communicate with, particularly when communicating with someone unfamiliar to them $[10,11]$. These communication difficulties can sometimes be very obstructive when trying to acquire information from a child about sensitive or emotionally provocative events. Establishing if robots could be used to bridge this gap and help facilitate communication may assist local authorities such as police and social services safeguard children that are in potentially unsafe environments. Using robots in this context for children with special needs is a logical step in light of the positive research indicating that children with special needs such as autism do respond well to robots in other contexts [3-5].

To investigate how children with special needs respond to a robot compared to a human interviewer, we interviewed 5 children twice (once by a robot, once by a human) in a counterbalanced study. The primary units of analysis for this study were: word count, information disclosure, time talking, and perceived engagement. These measures allowed us to compare the performance of the robotic interviewer with the human interviewer.

\section{Background}

The various sub domains of social robotics investigate the wide and diverse applications where social robots could be utilised, from robotic pets and educational aids $[1,2,12]$ to therapeutic and assistive tools $[4,13,14]$, research in social robotics indicates that both neurotypical children and children with special needs often show enthusiasm towards interacting with social robots [3, 15]. Given that children are often keen to interact with robots, investigating the possibility of robot mediation in an interview setting would be a logical step.

Interviewing children is often a very sensitive and delicate task that is carried out by skilled individuals that have undergone extensive training to perform such a skilled role. One area where the way in which interviews are conducted is particularly well researched and documented is within the police force. The UK police force often refers to a document called Achieving Best Evidence in Criminal Proceedings (ABE) [16]. The ABE was drafted for the UK's Home Office by a team of experts from varying backgrounds including psychology, law and social services. This document provides an effective structured and standardised method for interviewing young children which we adhered to as closely as possible where relevant.

The ABE suggests that interviews should have four phases, an establishing a rapport phase, a free narrative recall phase, a more specific questioning phase, and a closure phase. In the establishing a rapport phase simple non-invasive, topic-neutral 
questions are asked as well as the ground rules being set. In the free narrative recall phase the child is encouraged to recall as much information as possible without prompting, as this is thought to be the most reliable and accurate information. The question asking phase builds on the free narrative recall phase by asking more specific questions relating to the information gathered in the free narrative recall. It is important that the questions do not lead the interviewee as this could lead to misinformation. In the closing phase the topic returns to a neutral theme before thanking the child for their time and concluding the interview. In our study we used a similar structure but combined the free narrative phase with the questioning phase as we were investigating the relationship between question difficulty and how the child responded to the different interviewers.

When interviewing children it is important to remain neutral so as not to lead them towards a particular response or nonresponse. Maintain ones composure without subtly and unintentionally indicating thoughts and feelings can be difficult, particularly when interviewing children about a stressful or traumatic ordeal. The 2011 ABE states "the interviewer should not display surprise at information as this could be taken as a sign that the information is incorrect" [16]. This level of selfdiscipline can be quite difficult even for an experienced human interviewer. In addition to this it is also important that the interviewer does not appear to assume that someone is guilty "So far as possible, the interview should be conducted in a 'neutral' atmosphere, with the interviewer taking care not to assume, or appear to assume, the guilt of an individual whose alleged conduct may be the subject of the interview" [16]. More recent research also indicated that gestures can play a significant role in leading a witness [17]. Small gestures such as head nods can affect what someone will say in an interview [17]. Using a robot to interview a child could eliminate any of the subtle unintentional signs in body language that a human interviewer may give away, while the body language of the robot can be fully and precisely controlled by the interviewer.

Jones et al. [18] conducted a systematic review of 17 papers and concluded that children with a disability are up to four times more likely to be a victim of abuse than children without disabilities, however, the number of cases that result in prosecution is relatively low [19, 20]. Interviewing children with special needs can be very difficult, particularly when talking about a sensitive or emotionally provocative topic or event. A Mencap review states that "Those who can't communicate can't tell, and those who can't communicate well won't be believed" [21]. Research has shown that children with special needs such as autism are often very keen and willing to interact with robots [22, 23]. Robots such as KASPAR have been used for therapeutic purposes successfully on many occasions and have encouraged some of these children to be less isolated and more socially interactive [3, 4]. We hypothesis that these positive responses could possibly be replicated in an interview scenario and may prove be advantageous when using a robot in an interview context.

The primary research questions in this study were:

1. Will information disclosure differ between the two conditions? 
2. Do the quantitative aspects of the children's responses change when being interviewed by a robot?

3. Are there any differences in the temporal aspects of the verbal children's responses?

4. Will there be a difference in the children's engagement change dependent upon the interview partner?

In light of finding from previous studies we would expect to see a clear preference towards the robotic interview partner, although this may not affect the amount of information they provide in this particular study. We predict that the children will have a particularly clear preference to the robot with regards to how engaged they are with the interview partner and activity.

\section{$3 \quad$ Method}

\subsection{Participants}

This study took place in a UK primary school that specialises in catering for children with special needs. Five ( 3 male, 2 female) children took part in this study, each of which had different special need that included: Autism, BESD (Behavioural, Emotional and Social Difficulties), MLD (Moderate Learning Difficulties), and SLCN (Speech, Language and Communication Needs). The children were between the ages of 9 and 11, and some had interacted with KASPAR in previous studies in a different context.

\subsection{Procedure}

The children received a group introduction to both KASPAR the robot (figure 2) and the human interviewer at the school before the interviews commenced. The group introduction was used to familiarise the children with the interviewers and explain the procedure to them. Each child had 2 interviews in total, 1 with the robot and 1 with the human interviewer. The interviews were carried out in a 2 phase counterbalanced structure to minimise any crossover effects, with a 1 day gap between each interview. After each interview the child was asked some questions about the experience, in particular the interviewer. Once all of the interviews had been completed, the children were rewarded with some time to play with KASPAR along with a selection of other robotic toys.

The interviews followed a structure recommended by the ABE [16] of an easy topic neutral followed by a free narrative and questioning phase that focused on the main topic of interest. The interviews then returned to a neutral topic before concluding. The main theme and topic of the interviews focused on animals and pets. This topic was chosen because it offered sufficient scope for various different types and difficulty of questions. 


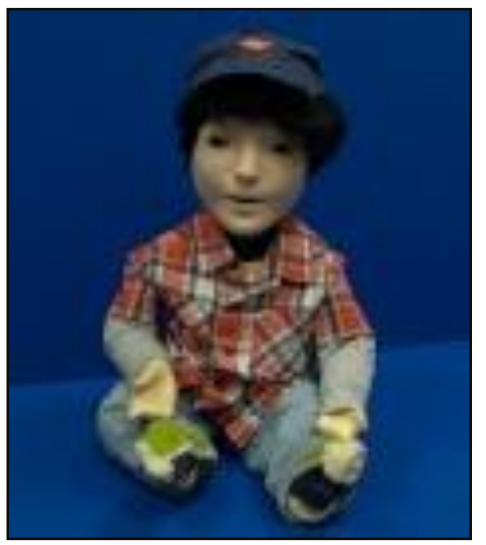

Fig. 1. KASPAR Robot.

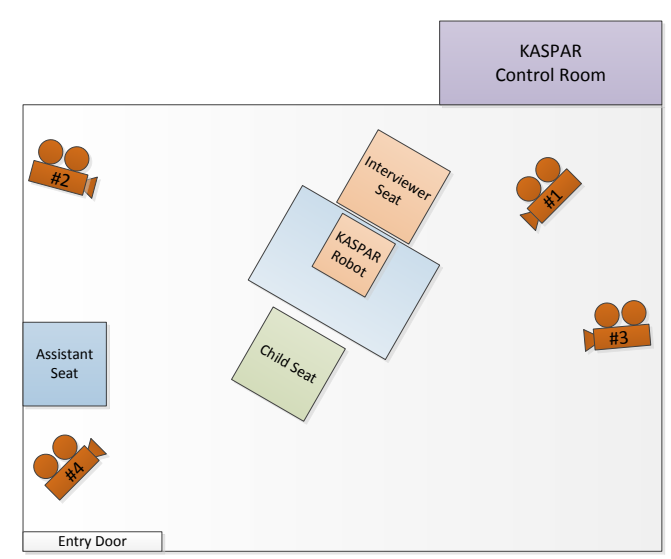

Fig. 2. Room layout.

\subsection{The setup}

The interviews were conducted in an unused classroom that contained a small lockable cupboard that the children were unable to see into. The cupboard was used as a control room for KASPAR and housed a monitor with a wireless connection to camera \#1 to observe the situation and make KASPAR respond appropriately (see Figure 2). The children were unaware that KASPAR was being controlled by a human triggering the correct questions and responses from a pre-recorded list. The interviews were led by the lead investigator either in person or remotely via KASPAR to help maintain consistency between the interviews. The children were escorted to and from the interviews by a second researcher that was unknown to the children. The second researcher remained in the room throughout the interviews, but was as non-reactive as possible. After each interview the children were asked by the second researcher to rate particular aspects of the experience, in particular the interaction partner.

\section{Measures}

There were four primary units of analysis in this study:

Communicative content - Communicative content refers to the statistical analysis of both the number of words spoken and the amount of qualitative pieces of information gathered from the children. All interviews were fully transcribed and analysed in detail for:

- Word count: words spoken throughout the interview excluding filler words.

- Filler word count: filler words such as "err", "errm", "hum".

- Keyword count: specific words related to questions e.g. "animal", "feed", "wild".

- Key point count: specific pieces of information relating to the questions asked. 
Some of these categories were also analysed proportionately where appropriate.

Duration analysis - The video footage was analysed and coded using the Observer XT software to measure the durations and specific temporal aspects of the interviews. The aspects we analysed in particular were:

- Interview duration: Full duration of the interview, start to finish.

- Child speaking duration: Total amount of time the child spent speaking.

- Child pause duration: Time child pauses for 2 seconds or more whilst speaking.

- Interviewer speaking duration: Total amount of time the interviewer speaks.

- Response time $\mathbf{C}>\mathbf{I}$ : Total amount of time between the child speaking and the interviewer responding.

- Response time I>C: Total amount of time between the interviewer speaking and the child responding.

Questionnaires - Immediately after each interview the child was asked to rate specific aspects of the experience:

- Interest: How interesting the child found the experience.

- Difficulty: How difficult the child found the interview.

- Fun: How much fun the child had participating.

- Duration: How long the child thought the interview took.

Perceived Engagement - In this study we analysed how engaged the children appeared to be in the activity and interaction partner. The engagement was coded by 3 independent blind coders that had no prior knowledge of the study or this line of research. The coders worked individually and were unknown to each other. Each coder viewed all of the videos start to finish before commencing the coding to acquire some familiarity for the children and their behaviour. This was an important phase because accurately assessing a child's engagement relies on having some previous knowledge of their full repertoire of behaviour within that particular context. The videos had been edited so that there was no audio and a portion of the screen had been obscured to hide the interaction partner from the coders. The coders were advised of where an interaction partner was located to order to assist in coding the engagement, but were not provided with any details about the experiment, as this may have adversely altered and biased their coding. The videos were coded with a simple on screen slider that moved with the position of the courser. A smile-meter placed in the top right hand corner of the screen changed according to the position of the slider. If the slider was towards the top this would indicate more engaged and the smile-meter would have a greater smile. If the slider was lower down the screen this would indicate less engaged and the smile-meter would change to more of a frown (Figure 3 ). This approach of continuous monitoring was originally employed for market research [24], but has also been adopted and adapted for measuring levels of fun and engagement for HRI and HCI applications [25, 26]. The method used in our study takes inspiration from these approaches, but uses a slightly different implementation to suite the requirements of our needs for this particular type of study. 


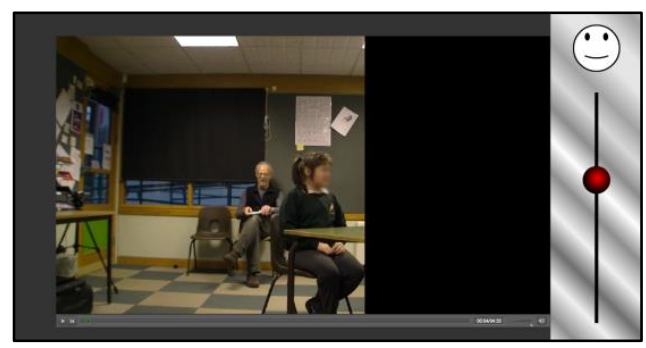

Fig. 3. Engagement coding program

The coders were given brief instructions on what may constitute engagement. However, it was ultimately left to the coder to decide what they personally considered to be engagement. The coders would move the slider up and down on a touch screen according to how engaged they personally felt that the child was in the interaction partner. The coding occurred at the normal video running speed and the program logged where the slider was on the screen in relation to the position of the video. The program logged the position of the slider 10 times every second, and all occurred in one continuous run with no pausing or rewinding.

Typically developing people are often very good at assessing how engaged someone is in an activity or an interaction partner. Rather than trying to code specific behaviours in great detail such as eye contact or body gestures, we tried this more subjective method and combined the results of each coder to establish an overall average for the engagement in each interview.

\section{$5 \quad$ Results}

The results from this study in Table 1 are consistent with previous findings in studies with typically developing children [6, 7, 9]. The only significant difference found relating to the perception of the children was how long the children thought the interviews took. The children thought the interviews with the robot were longer which was often correct as the results indicate that the mean duration of the interviews with KASPAR were longer than the human interviewer. Although not significant, it is indicated that on average the children found it more difficult to talking to KASPAR, this may be due to the text to speech voice of KASPAR being more difficult to understand. The quantitative results from this study are not significant, however, the qualitative results from looking at the interactions in the 2 conditions would indicate that some of the children seemed to be more engaged with the robot than the human interviewer. From the 5 children that took part in the study it appears that the children with the most severe communication difficulties seem a lot more engaged with the robot than the human interviewer. For example, three of the children's engagement scores were on average 108.99, 47.20 and 44.65 higher with KASPAR than the human interviewer. All three of these children had SLCN (Speech, Language and Communication Needs) and two of the children had Autism. 
Table 1. Quantitative comparison measures

\begin{tabular}{lccccc}
\hline \multicolumn{1}{c}{ Measure } & $\begin{array}{c}\text { KASPAR } \\
\text { Mean }\end{array}$ & $\begin{array}{c}\text { Human } \\
\text { Mean }\end{array}$ & $\begin{array}{c}\text { Mean } \\
\text { Diff. }\end{array}$ & t(p) & $\begin{array}{c}\text { Standard } \\
\text { Dev. }\end{array}$ \\
\hline 1=boring - 5=interesting & 3.40 & 3.40 & 0.00 & $0.00(1.000)$ & 1.428 \\
1=hard - 5=easy & 3.20 & 4.00 & -0.80 & $1.21(0.242)$ & 1.625 \\
1=no fun - 5=fun & 4.60 & 3.80 & 0.80 & $1.21(0.242)$ & 1.249 \\
1=long time - 5=quick & 2.20 & 5.00 & -2.80 & $2.44(0.025)^{*}$ & 1.800 \\
Overall key points & 18.40 & 18.80 & -0.40 & $0.09(0.928)$ & 7.902 \\
All Key words & 22.40 & 18.40 & 4.00 & $0.58(0.568)$ & 10.032 \\
Proportionate all key words & 0.17 & 0.21 & -0.04 & $0.84(0.412)$ & 0.079 \\
Child word count & 155.80 & 103.20 & 52.60 & $0.84(0.410)$ & 100.019 \\
Proportionate word count & 1.06 & 0.57 & 0.48 & $1.01(0.161)$ & 0.766 \\
Proportionate filler word count & 0.07 & 0.05 & 0.02 & $0.85(0.404)$ & 0.049 \\
Interviewer word count & 157.60 & 180.80 & -23.20 & $2.44(0.024)^{*}$ & 17.069 \\
Interview duration & 328.65 & 260.78 & 328.65 & $1.55(0.137)$ & 64.817 \\
Child response duration & 108.88 & 79.02 & 108.88 & $0.72(0.480)$ & 50.824 \\
Interviewer response duration & 55.25 & 76.77 & 55.25 & $2.94(0.008)^{*}$ & 12.715 \\
Response time C>I & 93.83 & 34.76 & 93.83 & $2.19(0.040)^{*}$ & 39.454 \\
Response time I>C & 36.58 & 30.47 & 36.58 & $0.80(0.433)$ & 17.070 \\
Child pause duration & 29.90 & 14.51 & 29.90 & $1.52(0.145)$ & 17.038 \\
Perceived average engagement & 321.09 & 278.29 & 42.81 & $2.28(0.084)$ & 72.256 \\
\hline
\end{tabular}

\section{Discussion and Conclusion}

The majority of the quantitative results from this study were consistent with the results of previous studies. The children interacted with the robot in a similar manner to which they did with a human interviewer. The information the amount and details the children revealed to the robot was similar to what they revealed to the human interviewer. However, the results of the engagement coding seem to suggest that in some cases the children seemed to be more engaged in the interaction with the robot than the human interviewer. Engagement is an important factor in any interaction, and the more engaged a child is in an activity, often the better their participation, which in an interview setting could be very beneficial. From a qualitative perspective the children were all very different and the differences in the interactions where varied. Some of the children had a very obvious preference for the KASPAR, which was reflected in the engagement coding, whilst others responded in a very similar manner in both interviews. It would appear that KASPAR seemed to benefit the children with the more severe conditions the most.

\subsection{Limitations and Future studies}

This study was a small case study with just 5 children, had there been more children participating in the study the results may have been clearer. The children in the study were individuals with various different needs and conditions, therefore the results of this study cannot be generalised to a population with particular condition as there is currently insufficient evidence. The children in this study were from one school in one geographical location covering and age range from 8 to 11 , children 
from other locations and age ranges may respond differently to a robot in this setting as age and cultural differences may have affected the way the children would respond in the interviews. The results from multiple studies have consistently shown that children do respond to a robot in an interview context. In futures studies we would like to create a more flexible user friendly system and put it in the hands of a professional interviewer to assess if this approach would be useful in a real world setting, however, it is important to first conduct studies in a less sensitive setting.

\section{Acknowledgements}

The authors would like to thank Ben Robins for providing assistance during the experiments and Maria Jesus Hervas Garcia, Rachel Marr-Johnson, Nicholas Shipp and Kinga Grof for help with the engagement and reliability coding. We would also like to thank the staff and children of the school who participated in this work.

\section{References}

1. Yoshida, S., D. Sakamoto, Y. Sugiura, M. Inami,T. Igarashi. RoboJockey: robotic dance entertainment for all. in SIGGRAPH Asia 2012 Emerging Technologies. 2012: ACM.

2. Tanaka, F.,M. Ghosh. The implementation of care-receiving robot at an English learning school for children. in HRI '11: Proceedings of the 6th international conference on Humanrobot interaction. 2011. Lausanne, Switzerland.

3. Wainer, J., K. Dautenhahn, B. Robins,F. Amirabdollahian. Collaborating with Kaspar: Using an autonomous humanoid robot to foster cooperative dyadic play among children with autism. in Humanoid Robots (Humanoids), 2010 10th IEEE-RAS International Conference on. 2010.

4. Robins, B., K. Dautenhahn,P. Dickerson. From isolation to communication: A case study evaluation of robot assisted play for children with autism with a minimally expressive humanoid robot. in Proc. the Second International Conferences on Advances in ComputerHuman Interactions, ACHI 09. 2009. Cancun, Mexico: IEEE Computer Society Press.

5. Huskens, B., R. Verschuur, J. Gillesen, R. Didden,E. Barakova, Promoting question-asking in school-aged children with autism spectrum disorders: Effectiveness of a robot intervention compared to a human-trainer intervention. Developmental neurorehabilitation, 2013(0): p. 1-12.

6. Bethel, C.L., M.R. Stevenson,B. Scassellati. Sharing a Secret: Interactions Between a Child, Robot, and Adult. in Presented at the Children with Robots Workshop at the 6th ACM/IEEE International Conference on Human-Robot Interaction. 2011. Lausanne, Switzerland.

7. Wood, L.J., K. Dautenhahn, A. Rainer, B. Robins, H. Lehmann,D.S. Syrdal, RobotMediated Interviews-How Effective Is a Humanoid Robot as a Tool for Interviewing Young Children? PLOS ONE, 2013. 8(3): p. e59448.

8. Bethel, C.L., D.K. Eakin, S. Anreddy, J.K. Stuart,D. Carruth. Eyewitnesses are misled by human but not robot interviewers. in Proceedings of the 8th ACM/IEEE international conference on Human-robot interaction. 2013: IEEE Press. 
9. Wood, L.J., K. Dautenhahn, H. Lehmann, B. Robins, A. Rainer,D.S. Syrdal, RobotMediated Interviews: Does a robotic interviewer impact question difficulty and information recovery?, in 12th European AAATE Conference. 2013: Vilamoura, Portugal.

10. Wing, L., The autistic spectrum: A guide for parents and professionals. 1996: Constable.

11. Jordan, R., Autistic spectrum disorders: an introductory handbook for practitioners. 1999: David Fulton Publishers London.

12. Barlett, B., V. Estivill-Castro,S. Seymon, Dogs or robots-Why do children see them as robotic pets rather than canine machines?, in 5th Australasian User Interface Conference (AUIC2004). 2004: Dunedin.

13. Goris, K., J. Saldien,D. Lefeber. Probo: a testbed for human robot interaction. in HRI '09: Proceedings of the 4th ACM/IEEE international conference on Human robot interaction. 2009. La Jolla, California, USA.

14. Kozima, H., M. Michalowski,C. Nakagawa, Keepon: A Playful Robot for Research, Therapy, and Entertainment. International Journal of Social Robotics, 2009. 1(1): p. 3-18.

15. Michalowski, M.P., S. Sabanovic,P. Michel, Roillo: Creating a Social Robot for Playrooms, in Robot and Human Interactive Communication, The 15th IEEE International Symposium, ROMAN. 2006.

16. UK Government, Achieving Best Evidence in Criminal Proceedings: Guidance on Interviewing Victims and Witnesses, and Using Special Measures. 2007 \& 2011, Home Office Criminal Justice System.

17. Gurney, D., K. Pine,R. Wiseman, The gestural misinformation effect : skewing eyewitness testimony through gesture. American Journal of Psychology, 2013.

18. Jones, L., M.A. Bellis, S. Wood, K. Hughes, E. McCoy, L. Eckley, G. Bates, C. Mikton, T. Shakespeare,A. Officer, Prevalence and risk of violence against children with disabilities: a systematic review and meta-analysis of observational studies. The Lancet, 2012.

19. Turk, V.,H. Brown, THE SEXUAL ABUSE OF ADULTS WITH LEARNING DISABILITES: RESULTS OF A TWO YEAR INCIDENCE SURVEY. Mental Handicap Research, 1993. 6(3): p. 193-216.

20. McCarthy, M.,D. Thompson, A prevalence study of sexual abuse of adults with intellectual disabilities referred for sex education. Journal of Applied Research in Intellectual Disabilities, 1997. 10(2): p. 105-124.

21. Mencap, Submission to the Sex Offences Review. 1999.

22. Robins, B., K. Dautenhahn, R. Boekhorst,A. Billard, Robotic assistants in therapy and education of children with autism: can a small humanoid robot help encourage social interaction skills? Universal Access in the Information Society, 2005. 4(2): p. 105-120.

23. Robins, B., K. Dautenhahn,P. Dickerson, Embodiment and Cognitive Learning-Can a Humanoid Robot Help Children with Autism to Learn about Tactile Social Behaviour?, in Social Robotics. 2012, Springer. p. 66-75.

24. Fenwick, I.,M.D. Rice, Reliability of continuous measurement copy-testing methods. Journal of Advertising Research, 1991.

25. Tanaka, F., A. Cicourel,J.R. Movellan, Socialization between toddlers and robots at an early childhood education center. Proceedings of the National Academy of Sciences, 2007. 104(46): p. 17954-17958.

26. Read, J., S. MacFarlane,C. Casey. Endurability, engagement and expectations: Measuring children's fun. in Interaction Design and Children. 2002: Shaker Publishing Eindhoven. 